\title{
Research on Integrated Management of Large-scale Engineering Construction Projects from the Perspective of Complex Systems
}

\author{
Peipei Liang \\ Guangdong College of Business and Technology, Zhaoqing, Guangdong, 526040
}

\begin{abstract}
Keywords: Integrated Management, Large-scale Engineering Construction Projects
\end{abstract}
\begin{abstract}
This paper analyzes the profound impact of the rapid development of modern information technology on the management of engineering construction projects and the problems existing in the management of large-scale engineering construction projects. On this basis, based on the system science, organizational behavior and complexity research, and based on the advanced management ideas and methods of other industries, the integrated management research of large-scale engineering construction projects based on modern information technology is carried out. The concept of comprehensive, whole process and integrated management of large-scale engineering construction projects is proposed, and an integrated management model for large-scale engineering construction projects based on modern information technology is established.
\end{abstract}

\section{Introduction}

The project construction project is the carrier of the country's fixed asset investment and an important channel for adjusting the product structure and industrial structure. China's Three Gorges Water Control Project, West-East Gas Transmission Project, Hong Kong International Airport, Beijing-Kowloon Railway, etc. have been completed one after another; the "five vertical and one horizontal” expressway network, high-speed railway, and giant port construction have been started or have been included in the new Century grand plan blueprint. Whether the objectives of these large-scale engineering construction projects, which are the leaders of engineering construction projects, can be realized on schedule, become the key to the economic and social benefits of project construction projects and the promotion of national economic growth.

Due to the huge impact of modern information technology, the ideas, organization, methods and means of engineering construction project management have undergone profound changes. The rapid development of modern information technology, especially computer network technology, has expanded the implementation space of the project, making it possible to implement large-scale engineering construction projects with many sub-projects across regions. In addition, developing countries have greater demand for large-scale infrastructure construction projects. The investment entities in the global engineering construction market tend to be diversified, the financial service system is improving day by day, various complex process technologies are becoming more and more perfect, and modern project management theory is developing rapidly. The trend of large-scale engineering construction projects is becoming more and more obvious. Large-scale engineering construction projects have huge investment, complex technology, long construction period, multiple construction sites covering multiple areas, and many sub-projects. It is a complex system engineering, and its management is very difficult. However, the shortcomings in the management of traditional engineering construction projects are more prominent in the management of large-scale engineering construction projects. This is mainly focused on issues such as backward project information management, separation of implementation processes, organizational and contract management models that cannot meet the requirements of the new situation, and inconsistent goals of the project participants. Therefore, we will absorb advanced management ideas, learn from the mature experience of other industries, and deeply understand the impact of modern information technology on large-scale engineering construction project owners and contracted enterprises and their project management models and thinking, and carry out large-scale engineering construction projects based on modern information technology. Integrated management research has great 
theoretical and practical significance.

\section{Status and characteristics of large-scale public construction projects}

Due to the continuous development of modern information technology, the organization and method requirements related to construction project management are getting higher and higher. Developing countries have great demand for large-scale public construction projects. With the diversification of investment entities in the global engineering construction market, the complexity of technological processes is gradually improved, and the project management theory is gradually improved. (1) Planned. The construction of large-scale public projects must be reviewed and approved by relevant state departments. During the project implementation process, all participants of the project must follow the relevant national regulations. Without the approval of the relevant units, the construction unit is not allowed to change the construction scale at will (2) the degree of collaboration is high. Large-scale public construction projects involve a wide range of issues, requiring close cooperation between various parties, timely communication and exchange, and joint completion of large-scale public construction projects. No matter which project stage has problems, it may affect the final goal and overall benefits of the project. (3) The construction period is long. Due to the large scale of large-scale public construction projects, complex technical processes, difficult management, and multiple majors, the construction period is long.

\section{Insufficient engineering management of traditional construction projects}

The project information management is the main reason for the backward management of engineering construction projects. If the project information management is not timely, information will not be timely and effectively communicated to the participants. The construction period of large-scale public construction projects is long, the project participants are numerous, and construction technology Complexity and other characteristics have determined that information management is a top priority. The participants in the traditional construction project have less communication and communication. Therefore, the information at each stage of the project cannot be transmitted and communicated in a timely manner. The information cannot be standardized and integrated. Some information cannot be found in time when it is used. This has caused a lot of inconvenience, which will cause problems such as reduced efficiency and long construction period of the project.

The pre-determination, design project management and construction project management in the traditional management mode are managed independently by the construction unit, design unit and construction unit, and the management contents are divided. There is no good communication at each stage. For the management content and management objectives of the company, the various stages can not be effectively connected. For example, only the owners and experts participate in the decision-making stage, and only the decision-making stage can make correct judgments and decisions, and the project construction stage and operation stage are not systematically implemented. Integration considerations; the design phase cannot be designed according to the specific construction conditions. After the design is completed, the contractor is selected to carry out the engineering construction, which causes many problems in the design to be not discovered in time, which leads to an increase in design changes, an increase in workload, and a delay in project construction progress. The separation of the various stages of the project will lead to a series of problems, such as the discontinuity of project management, the lack of unified planning and integrity of the entire construction project, and these problems are difficult to apply in large-scale public construction projects.

The participants in the traditional construction project divide the whole process of the project into various stages, and regard the parties as conflicting interests. Each participant is only responsible for the engineering objectives of their own stage, and does not affect the various objectives of the project as mutual influence. The unity of the unit is to be seen, the various objectives are carried out separately, and the timely communication and communication cannot be 
carried out during the construction process, which is not conducive to giving full play to the initiative and enthusiasm of the project participants, and the optimization of the objectives of the project construction project is difficult to achieve.

\section{Integrated management of environmental elements in large-scale engineering projects}

External environments are external forces and institutions that can potentially impact the performance of large engineering projects. The external factors are composed of two parts, namely the specific environment and the general environment. Large engineering projects The external environment is the sum of all external factors that affect the project, and it is in an environment that is constantly changing rapidly.

Specific environment. The specific environment includes elements that have a direct impact on the project management team's decisions and actions and are directly related to the achievement of the project objectives. The specific environmental factors faced by different projects are different, mainly including owners, government departments, participants, and the public and media.

General environment. The general environment includes: 1 social-economic-sustainability of environmental impact; 2 cultural influences; 3 standards and rules. The impact of the external environment on the project. The external environment has a major impact on the project, mainly reflected in: 1 The external environment determines the demand for the project and determines the existence value of the project; 2 The external environment determines the project technical plan and implementation plan and their optimization; 3 The environment is the risk The root of it.

The internal environment of large-scale project management refers to the specific resources and corresponding capabilities within the project, mainly including the project organization and its structure, project management team, project information, project culture and other elements.

Project organization and its structure. Think of large-scale project management as a system that includes system structure patterns, division of labor, and workflow.

Project management team. The Large Project Management Team consists of project managers and other members of the project team and is the organization directly responsible for managing the project. Under the leadership of the project manager, the project management team is guided by the overall objectives of the project, ensuring that all the work of the project is completed on time and in good quality within the budget, meeting the requirements of the owners and relevant government departments.

Project information. The information flow in the project includes two of the most important information exchange processes. The first is the exchange of information between the project and the outside world, including information input by the outside world and information exported by the project to the outside world. Second, the exchange of information within the project mainly includes top-down information flow, bottom-up information flow and horizontal Or network traffic.

Project culture. The culture of large-scale engineering projects refers to the unique leadership style, management methods, work level, membership quality, member beliefs, values and ideology of the project. It is the common values, identity, behavioral norms and organizational atmosphere of the project, and is the integration of the internal environment of the project. which performed.

\section{Main contents of integrated management of large-scale public construction projects}

The project construction project mainly includes three major objectives: quality target, construction period goal and cost target. The three goals are mutually interconnected and mutually constrained. The goal of integrated management of large-scale public construction projects is to achieve the goal by integrating all project objectives. Equilibrium, for example, when pursuing cost targets, it is not necessary to reduce the quality of the project and delay the construction period; when pursuing the project goal, it should not excessively increase the cost and reduce the quality of the project, prevent the project from pursuing a single goal in the implementation process, ignore the overall goal, and finally achieve the goal. The overall effect of the system.

The whole process of the project includes project decision-making, design, construction and 
putting into use. The whole process of integrated management of large-scale public construction projects is to effectively combine the various stages of the project, which can improve the working efficiency of the construction project, such as the most common problems in construction, due to the design. The process does not involve the actual situation in the construction, resulting in design changes, the construction period is postponed. If the design phase and the construction phase are integrated, the construction party participates in the design phase. Due to the construction party's understanding of the construction site and construction experience, the design unit can be reasonably effective. The proposal to reduce design changes during the construction process, reduce the corresponding workload, and reduce unnecessary delays in construction time. In order to achieve the optimization of large-scale public construction projects, it is necessary to carry out long-term planning throughout the whole process of the project, and achieve the best match between them through the integrated management of each stage.

As we all know, information management plays an important role in engineering projects. By collecting and integrating engineering information, it can improve work efficiency and reduce engineering costs due to communication problems. Through project information integration management, all participants can use information resources to realize project information data sharing and achieve project goals together; it is beneficial to the timely and effective communication of all parties involved, and avoid information transmission caused by improper communication. Missing or wrong situations; decision makers can grasp the correct information in time to avoid making wrong decisions and affect engineering projects.

\section{Conclusion}

Integration is the way in which some isolated elements or things are linked in some way to form an organic whole. Integrated management is to highlight the whole, use the concept of integration to link the various stages, guide and manage the construction project. The integrated management mode of large-scale public construction projects is guided by the integration of ideas, based on the characteristics of large-scale public construction projects and project management, through the combination of qualitative analysis and quantitative analysis, scientific project management methods, the integrated management model is well applied to the entire large-scale Public construction project management activities.

\section{References}

[1] Liu Yanhua. Research on the Integration Management of Public Projects [J]. Journal of Xi'an University of Architecture and Technology. 2012(5): 42-45

[2] Chen Yongqiang. Integrated Management of Large-scale Engineering Construction Projects [J].Journal of Tianjin University.2008 (3):202-205

[3] Zhao Likun. Research on Integrated Integrated Management Model of Engineering Projects [D]. Hebei University of Technology. 2003

[4] Li Mingrui. Research on Integrated Management Mode of Construction Engineering Project Based on BIM Technology [D]. Nanjing Forestry University. 2015

[5] Zhang Guozong. Research on integrated management mode of large-scale public welfare construction projects [J]. Technology Economics and Management Research, 2009 (8): 47-50. 